\title{
Lattice uniformities on effect algebras
}

\author{
Anna Avallone and Paolo Vitolo \\ Dipartimento di Matematica, Università della Basilicata \\ Contrada Macchia Romana, 85100 Potenza, Italy \\ avallone@unibas.it, vitolo@unibas.it
}

\begin{abstract}
Let $L$ be a lattice ordered effect algebra. We prove that the lattice uniformities on $L$ which make uniformly continuous the operations $\ominus$ and $\oplus$ of $L$ are uniquely determined by their system of neighbourhoods of 0 and form a distributive lattice. Moreover we prove that every such uniformity is generated by a family of weakly subadditive $[0,+\infty]$-valued functions on $L$.
\end{abstract}

Keywords Effect algebras, lattice uniformities, submeasures.

\section{Introduction}

Effect algebras have been introduced by D. J. Foulis and M. K. Bennett in 1994 [8] for modelling unsharp measurement in a quantum mechanical system. They are a generalization of many structures which arise in quantum physics (see [7]) and in Mathematical Economics (see [11, 9]), in particular of orthomodular lattices in non-commutative measure theory and MV-algebras in fuzzy measure theory. After 1994, there have been a great number of papers concerning effect algebras (see [10] for a bibliography).

In this paper we study D-uniformities on a lattice ordered effect algebra $L$, i.e. lattice uniformities on $L$ which makes uniformly continuous the operations $\ominus$ and $\oplus$ of $L$.

Starting point of our paper is observing the key role played by D-uniformities in the study of modular measures on $L$ (see $[1,2,3]$ ), since every modular measure on $L$ generates a D-uniformity, and the role played in the study of modular functions on orthomodular lattices (see [16]) and of measures on MV-algebras (see $[6,13]$ ) by the lattice structure of filters which generate lattice uniformities making uniformly continuous the operations of these structures.

In the first part of the paper, we give a description of the filters which are systems of neighbourhoods of 0 in D-uniformities on $L$ - called D-filters - and we prove that there exists an order isomorphism between the lattice of all D-uniformities on $L$ and the lattice of all D-filters on $L$. In particular every D-uniformity is uniquely determined by its system of neighbourhoods of 0 . As a consequence, we obtain that the lattice of all D-uniformities on $L$ is distributive.

Our results extend similar results of [16] in orthomodular lattices (see also [5]) and of [6] and [13] in MV-algebras, and give as particular case the order isomorphism found in [4] between some lattice congruences and some lattice ideals.

In the second part of the paper, we apply the results of the first part to prove that every D-uniformity on $L$ is generated by a family of weakly subadditive $[0,+\infty]$-functions on $L$.

\section{Preliminaries}

An effect algebra [10] is a set $E$, with two distinguished elements 0 and 1 , and a partially defined operation $\oplus$ such that for all $a, b, c \in E$ :

(E1) If $a \oplus b$ is defined, then $b \oplus a$ is defined and $a \oplus b=b \oplus a$.

(E2) If $b \oplus c$ is defined and $a \oplus(b \oplus c)$ is defined, then $a \oplus b$ and $(a \oplus b) \oplus c$ are defined, and $a \oplus(b \oplus c)=(a \oplus b) \oplus c$.

(E3) There exists a unique $a^{\perp} \in E$ such that $a \oplus a^{\perp}$ is defined and $a \oplus a^{\perp}=1$.

(E4) If $a \oplus 1$ is defined, then $a=0$. 
It is easily seen that $a \oplus 0$ is always defined and equals $a$. If $a \oplus b$ is defined, we say that $a$ and $b$ are orthogonal and write $a \perp b$.

In an effect algebra $E$ another partially defined operation $\ominus$ can be defined by the following rule: $c \ominus a$ exists and equals $b$ if and only if $a \oplus b$ exists and equals $c$. In particular, $a^{\perp}=1 \ominus a$. Moreover, if $a \perp b$, then $a \oplus b=\left(a^{\perp} \ominus b\right)^{\perp}=\left(b^{\perp} \ominus a\right)^{\perp}$.

In an effect algebra $E$ a partial ordering relation $\leq$ can be defined as follows: $a \leq c$ if and only if, for some $b \in E, a \oplus b$ exists and equals $c$. Hence $c \ominus a$ is defined if and only if $a \leq c$. Moreover $a \perp b$ if and only if $a \leq b^{\perp}$.

If $a \vee b$ and $a \wedge b$ exist for all $a, b \in E$, then we say that $E$ is a lattice ordered effect algebra (otherwise called D-lattice). In this case, we define the symmetric difference of any two elements $a$ and $b$ in $E$ as $a \triangle b=(a \vee b) \ominus(a \wedge b)$.

Throughout the paper, the symbol $L$ will always denote a lattice ordered effect algebra. Let us recall that $L$ is an $M V$-algebra if and only if $(a \vee b) \ominus b=a \ominus(a \wedge b)$ for all $a, b \in L$, while $L$ is an orthomodular lattice if and only if $a^{\perp} \wedge a=0$ for every $a \in L$.

We will make use of the following properties (for the proofs we refer to [10]).

1.1. Proposition. For all $a, b, c \in L$ we have:

(i) If $a \leq b$, then $b \ominus a \leq b$ and $b \ominus(b \ominus a)=a$.

(ii) If $a \leq b \leq c$, then $c \ominus b \leq c \ominus a$ and $(c \ominus a) \ominus(c \ominus b)=b \ominus a$.

(iii) If $a \leq b \leq c$, then $b \ominus a \leq c \ominus a$ and $(c \ominus a) \ominus(b \ominus a)=c \ominus b$.

(iv) If $a \leq b^{\perp}$ and $a \oplus b \leq c$, then $c \ominus(a \oplus b)=(c \ominus a) \ominus b=(c \ominus b) \ominus a$.

(v) If $a \leq b \leq c^{\perp}$, then $a \oplus b \leq b \oplus c$ and $(b \oplus c) \ominus(a \oplus c)=b \ominus a$.

(vi) If $a \leq b \leq c$, then $a \oplus(c \ominus b)=c \ominus(b \ominus a)$.

(vii) If $a \leq b^{\perp} \leq c^{\perp}$, then $a \oplus(b \ominus c)=(a \oplus b) \ominus c$.

(viii) If $a \leq c$ and $b \leq c$, then $c \ominus(a \vee b)=(c \ominus a) \wedge(c \ominus b)$ and $c \ominus(a \wedge b)=(c \ominus a) \vee(c \ominus b)$.

(ix) If $c \leq a$ and $c \leq b$, then $(a \wedge b) \ominus c=(a \ominus c) \wedge(b \ominus c)$ and $(a \vee b) \ominus c=(a \ominus c) \vee(b \ominus c)$.

(x) If $a \leq c^{\perp}$ and $b \leq c^{\perp}$, then $(a \vee b) \oplus c=(a \oplus c) \vee(b \oplus c)$ and $(a \wedge b) \oplus c=(a \oplus c) \wedge(b \oplus c)$.

Let $\mathcal{U}$ be a uniformity on $L$. We say that $\mathcal{U}$ is a lattice uniformity [14] if the operations $\vee$ and $\wedge$ are uniformly continuous with respect to $\mathcal{U}$.

A D-uniformity [1] is a lattice uniformity which makes the operations $\oplus$ and $\ominus$ uniformly continuous, too. The set of all D-uniformities on $L$ will be denoted by $\mathcal{D} U(L)$. It is easy to see that $\mathcal{D U}(L)$ - ordered by inclusion - is a complete lattice, with the discrete uniformity and the trivial uniformity as greatest and smallest elements, respectively.

Given $U, V \subset L \times L$, we put

$$
\begin{aligned}
& U \vee V=\left\{\left(a_{1} \vee b_{1}, a_{2} \vee b_{2}\right):\left(a_{1}, a_{2}\right) \in U,\left(b_{1}, b_{2}\right) \in V\right\}, \\
& U \wedge V=\left\{\left(a_{1} \wedge b_{1}, a_{2} \wedge b_{2}\right):\left(a_{1}, a_{2}\right) \in U,\left(b_{1}, b_{2}\right) \in V\right\}, \\
& U \ominus V=\left\{\left(a_{1} \ominus b_{1}, a_{2} \ominus b_{2}\right): b_{1} \leq a_{1}, b_{2} \leq a_{2},\left(a_{1}, a_{2}\right) \in U,\left(b_{1}, b_{2}\right) \in V\right\} .
\end{aligned}
$$

It is known (see [14]) that a uniformity $\mathcal{U}$ on $L$ is a lattice uniformity if and only if for every $U \in \mathcal{U}$ there exist $V \in \mathcal{U}$ such that $V \vee \Delta \subset U$ and $V \wedge \Delta \subset U$, where $\Delta=\{(a, a): a \in L\}$.

Similarly, it has been shown in [1] that a lattice uniformity $U$ on $L$ is a D-uniformity if and only if for every $U \in \mathcal{U}$ there exist $V \in \mathcal{U}$ such that $V \ominus \Delta \subset U$ and $\Delta \ominus V \subset U$.

\section{D-uniformities and D-filters}

2.1. Definition. A filter $\mathcal{F}$ of subsets of a D-lattice $L$ is called $D$-filter if it satisfies the following:

(F1) $\forall F \in \mathcal{F} \quad \exists F^{\prime} \in \mathcal{F}: \quad \forall a, b \in F^{\prime} \quad[a \perp b \Longrightarrow a \oplus b \in F]$;

(F2) $\forall F \in \mathcal{F} \quad \exists G \in \mathcal{F}: \quad \forall a \in G \quad \forall c \in L \quad(a \vee c) \ominus c \in F$.

The set of all D-filters on $L$ will be denoted by $\mathcal{F N D}(L)$.

Note that, by 1.1(ii), a filter $\mathcal{F}$ satisfies (F2) if and only if, for every $F \in \mathcal{F}$, there exists $G \in \mathcal{F}$ such that, for all $a \in G$ and all $c \in L$, one has $c \ominus\left(a^{\perp} \wedge c\right) \in F$.

We shall prove, in Theorem 2.4 below, that $\mathcal{F N D}(L)$ is isomorphic to $\mathcal{D U}(L)$ and that $\mathcal{F}$ is a D-filter if and only if $\mathcal{F}$ is the system of neighbourhoods of 0 in a D-uniformity.

2.2. Lemma. For every $a, b, c, d \in L$ such that $c \leq a, c \leq b, d \geq a$ and $d \geq b$ one has $(a \ominus c) \triangle(b \ominus c)=a \triangle b=(d \ominus a) \triangle(d \ominus b)$. 
Proof. Indeed, applying 1.1(ix), and 1.1(iii), one gets $(a \ominus c) \triangle(b \ominus c)=((a \ominus c) \vee(b \ominus$ $c)) \ominus((a \ominus c) \wedge(b \ominus c))=((a \vee b) \ominus c) \ominus((a \wedge b) \ominus c)=(a \vee b) \ominus(a \wedge b)=a \triangle b$. Similarly, applying 1.1(viii), and 1.1(ii), one gets $(d \ominus a) \triangle(d \ominus b)=((d \ominus a) \vee(d \ominus b)) \ominus((d \ominus a) \wedge(d \ominus b))=$ $(d \ominus(a \wedge b)) \ominus(d \ominus(a \vee b))=(a \vee b) \ominus(a \wedge b)=a \triangle b$.

2.3. Proposition. A $D$-filter $\mathcal{F}$ on $L$ has the following properties:

(i) $\forall F \in \mathcal{F} \quad \exists G \in \mathcal{F}: \quad \forall a \in G \quad \forall b \in L \quad[b \leq a \Longrightarrow b \in F]$;

(ii) $\forall F \in \mathcal{F} \quad \exists G \in \mathcal{F}: \quad \forall a, b \in G \quad a \vee b \in F$;

(iii) $\forall F \in \mathcal{F} \quad \exists G \in \mathcal{F}: \quad \forall x, y, z \in L \quad[x \triangle y \in G \Longrightarrow(x \vee z) \triangle(y \vee z) \in F]$;

(iv) $\forall F \in \mathcal{F} \quad \exists G \in \mathcal{F}: \quad \forall x, y, z \in L \quad[x \triangle y \in G \Longrightarrow(x \wedge z) \triangle(y \wedge z) \in F]$;

(v) $\forall F \in \mathcal{F} \quad \exists G \in \mathcal{F}: \quad \forall x, y, z \in L \quad[x \triangle y \in G, y \triangle z \in G \Longrightarrow x \triangle z \in F]$.

Proof.

(i) Let $F \in \mathcal{F}$ and let $G \in \mathcal{F}$ such that (F2) is satisfied. Given any $a \in G$ and any $b \in L$ with $b \leq a$, put $c=a \ominus b$; then $b=a \ominus(a \ominus b)=(a \vee(a \ominus b)) \ominus(a \ominus b)=(a \vee c) \ominus c \in F$.

(ii) Given $F \in \mathcal{F}$, let $F^{\prime} \in \mathcal{F}$ satisfying (F1), and let $G \in \mathcal{F}$ satisfying (F2) with $F^{\prime}$ in place of $F$. If $a, b \in G$, then $(a \vee b) \ominus b \in F^{\prime}$; moreover $b \in F^{\prime}$ by (i): therefore $a \vee b=((a \vee b) \ominus b) \oplus b \in F$. (iii) Let $F \in \mathcal{F}$ and let $G \in \mathcal{F}$ such that (F2) is satisfied. Given $x, y, z$ such that $x \triangle y \in G$, we put $a=x \triangle y$ and $c=((x \vee z) \wedge(y \vee z)) \ominus(x \wedge y)$ and we show that $(x \vee z) \triangle(y \vee z)=(a \vee c) \ominus c$. First observe that $x \vee y \vee z=(x \vee y) \vee((x \vee z) \wedge(y \vee z))$; now, applying 1.1(x) and 1.1(v), we have:

$$
\begin{gathered}
(x \vee z) \triangle(y \vee z)=(x \vee y \vee z) \ominus((x \vee z) \wedge(y \vee z)) \\
=((x \vee y) \vee((x \vee z) \wedge(y \vee z))) \ominus((x \vee z) \wedge(y \vee z)) \\
=(((x \triangle y) \oplus(x \wedge y)) \vee((x \vee z) \wedge(y \vee z))) \ominus((x \vee z) \wedge(y \vee z)) \\
=((a \oplus(x \wedge y)) \vee(c \oplus(x \wedge y))) \ominus(c \oplus(x \wedge y)) \\
=((a \vee c) \oplus(x \wedge y)) \ominus(c \oplus(x \wedge y))=(a \vee c) \ominus c .
\end{gathered}
$$

(iv) Given $F \in \mathcal{F}$, take $G \in \mathcal{F}$ such that (iii) is satisfied, and let $x, y, z$ such that $x \triangle y \in G$. By Lemma 2.2 we have $x^{\perp} \triangle y^{\perp}=x \triangle y$, and therefore $(x \wedge z) \triangle(y \wedge z)=\left(x^{\perp} \vee z^{\perp}\right)^{\perp} \triangle\left(y^{\perp} \vee z^{\perp}\right)^{\perp}=$ $\left(x^{\perp} \vee z^{\perp}\right) \triangle\left(y^{\perp} \vee z^{\perp}\right) \in F$.

(v) Given $F \in \mathcal{F}$, let $F_{1} \in \mathcal{F}$ satisfy (ii), let $F_{2} \in \mathcal{F}$ satisfy (i) with $F_{1}$ in place of $F$, let $F_{3} \in \mathcal{F}$ satisfy $(\mathrm{F} 1)$ with $F_{2}$ in place of $F$ and let $G \in \mathcal{F}$ satisfy (iii) with $F_{3}$ in place of $F$. If $a, b, c \in L$ are such that both $x \triangle y$ and $x \triangle z$ belong to $G$, then $a=(x \vee y \vee z) \ominus(y \vee z)=$ $\left((x \vee(x \vee z)) \triangle(y \vee(y \vee z)) \in F_{3}\right.$ and $b=(y \vee z) \ominus z=(y \vee z) \triangle(z \vee z) \in F_{3}$ also. It follows that $(x \vee y \vee z) \ominus z=a \oplus b \in F_{2}$, so that $(x \vee z) \ominus z \in F_{1}$. Similarly one shows that $(x \vee z) \ominus x \in F_{1}$. Hence $x \triangle z=((x \vee z) \ominus z) \vee((x \vee z) \ominus x) \in F$.

\subsection{Theorem.}

(a) If $\mathcal{U}$ is a D-uniformity, then the filter $\mathcal{F}_{\mathcal{U}}$ of neighbourhoods of 0 in $\mathcal{U}$ is a D-filter.

(b) Let $\mathcal{F}$ be a $D$-filter and, for each $F \in \mathcal{F}$, let $F^{\triangle}=\{(a, b) \in L \times L: a \triangle b \in F\}$. Then $\mathcal{B}=\left\{F^{\triangle}: F \in \mathcal{F}\right\}$ is a base for a $D$-uniformity whose filter of neighbourhoods of 0 is $\mathcal{F}$.

(c) The mapping $\Psi: \mathcal{U} \mapsto \mathcal{F}_{\mathfrak{U}}$ is an order-isomorphism of $\mathcal{D U}(L)$ onto $\mathcal{F} \mathcal{N} \mathcal{D}(L)$ (both ordered by inclusion).

Proof.

(a) Since $\oplus$ is continuous at $(0,0)$, for every $F \in \mathcal{F}_{\mathfrak{u}}$ there exists $F^{\prime} \in \mathcal{F}_{\mathfrak{u}}$ such that if $(a, b) \in$ $F^{\prime} \times F^{\prime}$ and $a \perp b$, then $a \oplus b \in F$ : this gives (F1). To prove (F2), let $F \in \mathcal{F}_{\mathfrak{u}}$ and let $U \in \mathcal{U}$ with $U(0) \subseteq F$; by uniform continuity of $\ominus$ and $\vee$, there exist $V_{1}, V_{2} \in \mathcal{U}$ such that $V_{1} \ominus \Delta \subset U$ and $V_{2} \vee \Delta \subset V_{1}$. Now put $G=V_{2}(0)$, and consider any $a \in G$; then $(0, a) \in V_{2}$, so that for every $c \in L$ we have $(c, a \vee c) \in V_{2} \vee \Delta \subset V_{1}$ and hence $(0,(a \vee c) \ominus c) \in V_{1} \ominus \Delta \subset U$ which means that $(a \vee c) \ominus c \in U(0) \subseteq F$. 
(b) Clearly $F^{\triangle}$ is symmetric and $\Delta \subset F^{\triangle}$ for every $F \in \mathcal{F}$. Moreover, given $F_{1}, F_{2} \in \mathcal{F}$, let $F_{3}=\mathcal{F}_{1} \cap F_{2}$ : then $F_{3}^{\triangle}=F_{1}^{\triangle} \cap F_{2}^{\triangle}$. Finally, if $F \in \mathcal{F}$ and $G \in \mathcal{F}$ satisfies $2.3(\mathrm{v})$, we have that $G^{\triangle} \circ G^{\triangle} \subseteq F^{\triangle}$. Therefore $\mathcal{B}$ is a base for a uniformity $\mathcal{U}$.

Now fix $U \in \mathcal{U}$. We show that there exists $V \in \mathcal{U}$ such that both $V \vee \Delta$ and $V \wedge \Delta$ are contained in $U$. Let $G \in \mathcal{F}$ satisfy 2.3(iii) and put $V=G^{\triangle}$. Given $(x, y) \in V \vee \Delta$, take $a, b, c \in L$ with $x=a \vee c, y=b \vee c$ and $(a, b) \in V$ that is $a \triangle b \in G$; by 2.3(iii), we have $x \triangle y=(a \vee c) \triangle(b \vee c) \in F$, that is $(x, y) \in F^{\triangle}$ : we conclude that $V_{1} \vee \Delta \subset F^{\triangle} \subseteq U$; since the same $G$ also satifies 2.3(iv) one sees in a similar way that $V \wedge \Delta \subset F^{\triangle} \subseteq U$, too. Next, we show that there exists $V \in \mathcal{U}$ such that both $V \ominus \Delta$ and $\Delta \ominus V$ are contained in $U$. Choose $F \in \mathcal{F}$ such that $F^{\triangle} \subseteq U$ and put $V=F^{\triangle}$. By Lemma 2.2, one has $F^{\triangle} \ominus \Delta=\{(a \ominus c, b \ominus c): c \leq a, c \leq b, a \triangle b \in F\}=\{(a \ominus c, b \ominus c)$ : $c \leq a, c \leq b,(a \ominus c) \triangle(b \ominus c) \in F\}=F^{\triangle}$ and similarly one sees that $\Delta \ominus F^{\triangle}=F^{\triangle}$ : hence $V \ominus \Delta \subset U$ and $\Delta \ominus V \subset U$.

It remains to prove that the filter of neighbourhoods of 0 in $\mathcal{U}$ coincides with $\mathcal{F}$. First observe that, given any $F \in \mathcal{F}$, we have

$$
F^{\triangle}(0)=\left\{a \in L:(0, a) \in F^{\triangle}\right\}=\{a \in L: a \triangle 0 \in F\}=F
$$

and therefore $F$ is a neighbourhood of 0 in $\mathcal{U}$. Conversely, if $G$ is a neighbourhood of 0 in $\mathcal{U}$, since $\mathcal{B}$ is a base for $\mathcal{U}$, there exists $F \in \mathcal{F}$ such that $F^{\triangle}(0) \subseteq G$; by $(2 ; 1)$, this means that $F \subset G$ and hence $G \in \mathcal{F}$, because $\mathcal{F}$ is a filter.

(c) It follows from (a) that $\Psi$ maps $\mathcal{D} U(L)$ into $\mathcal{F N} \mathcal{D}(L)$. Now for any $\mathcal{F} \in \mathcal{F N} \mathcal{D}(L)$ let $\Phi(\mathcal{F})$ denote the D-uniformity constructed as in (b): since $\Psi(\Phi(\mathcal{F}))=\mathcal{F}$, we have that $\Psi$ is onto. Moreover if $\mathcal{F}_{1}, \mathcal{F}_{2} \in \mathcal{D U}(L)$ and $\mathcal{F}_{1} \subset \mathcal{F}_{2}$, then $\left\{F^{\triangle}: F \in \mathcal{F}_{1}\right\} \subseteq\left\{F^{\triangle}: F \in \mathcal{F}_{1}\right\}$ whence $\Phi\left(\mathcal{F}_{1}\right) \subseteq \Phi\left(\mathcal{F}_{2}\right)$. On the other hand, if $\mathcal{U}_{1}, \mathcal{U}_{2} \in \mathcal{D U}(L)$ and $\mathcal{U}_{1} \subset \mathcal{U}_{2}$, then the topology induced by $\mathcal{U}_{1}$ is coarser than the one induced by $\mathcal{U}_{2}$, hence $\Psi\left(\mathcal{U}_{1}\right) \subseteq \Psi\left(\mathcal{U}_{2}\right)$.

Finally we show that $\Phi=\Psi^{-1}$, so that $\Psi$ is one-to-one. Given $\mathcal{F} \in \mathcal{F} \mathcal{N} \mathcal{D}(L)$, we consider any $\mathcal{U} \in \mathcal{D} U(L)$ such that $\mathcal{F}=\Psi(\mathcal{U})$ and prove that $\Phi(\mathcal{F})=\mathcal{U}$. If $F \in \mathcal{F}$, then it is a neighbourhood of 0 , hence there is $U \in \mathcal{U}$ such that $U(0) \subseteq F$; by uniform continuity of $\triangle$, there exists $V \in \mathcal{U}$ with $V \triangle \Delta \subset U$. Now let $(a, b) \in V$ : we have $(0, a \triangle b)=(a \triangle a, b \triangle a) \in V \triangle \Delta \subset U$, whence $a \triangle b \in U(0) \subseteq F$. Hence $V \subset F^{\triangle}$ and therefore $\mathcal{U}$ is finer than $\Phi(\mathcal{F})$. Conversely let $U \in \mathcal{U}$; consider a symmetric $V_{1} \in \mathcal{U}$ with $V_{1} \circ V_{1} \subset U$, and take $V_{2}, V_{3} \in \mathcal{U}$ such that $V_{2} \vee \Delta \subset V_{1}$ and $V_{3} \oplus \Delta \subset V_{2}$. Put $F=V_{3}(0)$, so that $F \in \mathcal{F}$; if $(a, b) \in F^{\triangle}$, we have $a \triangle b \in F$, that is $(0, a \triangle b) \in V_{3}$. It follows that $(a \wedge b, a \vee b)=(0 \oplus(a \wedge b),(a \triangle b) \oplus(a \wedge b)) \in V_{3} \oplus \Delta \subset V_{2}$, hence $(a, a \vee b)=((a \wedge b) \vee a,(a \vee b) \vee a) \in V_{2} \vee \Delta \subset V_{1}$ and, similarly $(b, a \vee b) \in V_{1}$; since $V_{1}^{-1}=V_{1}$ we also have $(a \vee b, b) \in V$, and then $(a, b) \in V_{1} \circ V_{1} \subset U$ : therefore $F^{\triangle} \subseteq U$. We conclude that $\mathcal{U} \subset \Phi(\mathcal{F})$, whence the equality.

The reader should note that the above theorem implies, as particular cases, the results of $[6$, Theor. 2.1] and [13, Theor. 3.6] for MV-algebras, as well as [16, Theor. 1.1] for orthomodular lattices.

From Theorem 2.4(c), by restricting to principal filters, one can deduce the order isomorphism between D-congruences and D-ideals, which has been found, using a different approach, in [4, Theor. 4.5].

2.5. Proposition. Let $\mathcal{F}$ be the filter of neighbourhoods of 0 in a $D$-uniformity $\mathfrak{U}$; for every $F \in \mathcal{F}$, let $F^{\oplus}=\{(a, b) \in L \times L: \exists h, k \in F: h \perp a, k \perp b, a \oplus h=b \ominus k\}$ and $F^{\ominus}=\{(a, b) \in$ $L \times L: \exists i, j \in F: i \leq a, j \leq b, a \ominus i=b \ominus j\}$. Then both $\left\{F^{\oplus}: F \in \mathcal{F}\right\}$ and $\left\{F^{\ominus}: F \in \mathcal{F}\right\}$ are bases for $\mathcal{U}$.

Proof. It suffices to show that, for every $F \in \mathcal{F}$, there exist $F_{1}, F_{2} \in \mathcal{F}$ such that $F^{\oplus}, F^{\ominus} \supseteq F_{1}^{\triangle}$ and $F_{2}^{\oplus}, F_{2}^{\ominus} \subseteq F^{\triangle}$.

Let $F_{1} \in \mathcal{F}$ satisfy 2.3(i); given $(a, b) \in F_{1}^{\triangle}$, we put $h=(a \vee b) \ominus a, k=(a \vee b) \ominus b, i=a \ominus(a \wedge b)$ and $j=b \ominus(a \wedge b)$. Since $h \leq(a \vee b) \ominus(a \wedge b)=a \triangle b \in F_{1}$, we have $h \in F$; in the same way one sees that $k, i$ and $j$ belong to $F$, too. Moreover we have $a \oplus h=a \oplus((a \vee b) \ominus a)=$ 
$a \vee b=b \oplus((a \vee b) \ominus b)=b \oplus k$, so that $(a, b) \in F^{\oplus}$. Similarly, applying 1.1(i), we have $a \ominus i=a \ominus(a \ominus(a \wedge b))=a \wedge b=b \ominus(b \ominus(a \wedge b))=b \ominus j$, so that $(a, b) \in F^{\ominus}$.

Now let $G \in \mathcal{F}$ satisfy 2.3(ii), and take $F_{2} \in \mathcal{F}$ satisfying 2.3(i) with $G$ in place of $F$; given $(a, b) \in F_{2}^{\oplus}$, there are $h, k \in F_{2}$ such that $h \perp a, k \perp b$ and $a \oplus h=b \oplus k$. Since $a \vee b \leq$ $(a \oplus h) \vee(b \oplus k)=a \oplus h=b \oplus k$, we get $(a \vee b) \ominus a \leq h$ and $(a \vee b) \ominus b \leq k$, so that both $(a \vee b) \ominus a$ and $(a \vee b) \ominus b$ belong to $G$. By 1.1(viii), we have $a \triangle b=((a \vee b) \ominus a) \vee((a \vee b) \ominus b)$ hence $a \triangle b \in F$, i.e. $(a, b) \in F^{\triangle}$. Similarly, given $(a, b) \in F_{2}^{\ominus}$, take $i, j \in F_{2}$ such that $i \leq a, j \leq b$ and $a \ominus i=b \ominus j$; observe that $a \ominus i=(a \ominus i) \wedge(b \ominus j) \leq a \wedge b$ thus, applying 1.1(i), $i=a \ominus(a \ominus i) \geq a \ominus(a \wedge b)$. It follows that $a \ominus(a \wedge b) \in G$, and in the same way one sees that $b \ominus(a \wedge b) \in G$, too. By 1.1(ix), we have $a \triangle b=(a \ominus(a \wedge b)) \vee(b \ominus(a \wedge b))$ hence $a \triangle b \in F$, i.e. $(a, b) \in F^{\triangle}$.

Given $F, G \subset L$, we will put $F \oplus G=\{f \oplus g: f \perp g, f \in F, g \in G\}$. Using this notation, condition (F1) may be rewritten as follows: $\forall F \in \mathcal{F} \quad \exists F^{\prime} \in \mathcal{F}: \quad F^{\prime} \oplus F^{\prime} \subseteq F$.

\subsection{Proposition.}

(a) If $\mathcal{F}, \mathcal{G} \in \mathcal{F N D}(L)$, then $\{F \oplus G: F \in \mathcal{F}, G \in \mathcal{G}\}$ is a base for $\mathcal{F} \wedge \mathcal{G}$ in $\mathcal{F N D}(L)$.

(b) If $\Gamma \subset \mathcal{F N D}(L)$, then $\bigvee \Gamma$ in $\mathcal{F N D}(L)$ is the set of all intersections of finite subsets of $\bigcup \Gamma$. In particular $\mathcal{G}_{1} \vee \mathcal{G}_{2}=\left\{G_{1} \cap G_{2}: G_{1} \in \mathcal{G}_{1}, G_{2} \in \mathcal{G}_{2}\right\}$ for all $\mathcal{G}_{1}, \mathcal{G}_{2} \in \mathcal{F} \mathcal{N D}(L)$.

Proof.

(a) First observe that

$$
\forall F \in \mathcal{F} \quad \forall G \in \mathcal{G} \quad F \cup G \subset F \oplus G .
$$

Indeed, since $0 \in G$, one has $F=\{f \oplus 0: f \in F\} \subseteq\{f \oplus g: f \perp g, f \in F, g \in G\}=F \oplus G$, and similarly for $G$. In particular, all sets $F \oplus G$ with $F \in \mathcal{F}$ and $G \in \mathcal{G}$ are non-empty. Now, given $F_{1} \oplus G_{1}$ and $F_{2} \oplus G_{2}$, with $F_{1}, F_{2} \in \mathcal{F}$ and $G_{1}, G_{2} \in \mathcal{G}$, let $F=F_{1} \cap F_{2}$ and $G=G_{1} \cap G_{2}$. We have $F \oplus G=\{f \oplus g: f \perp g, f \in F, g \in G\} \subseteq\left\{f \oplus g: f \perp g, f \in F_{1}, g \in G_{1}\right\}=F_{1} \oplus G_{1}$ and, similarly, $F \oplus G \subset F_{2} \oplus G_{2}$ : hence $F \oplus G \subset\left(F_{1} \oplus G_{1}\right) \cap\left(F_{2} \oplus G_{2}\right)$. Therefore $\{F \oplus G: F \in \mathcal{F}, G \in \mathcal{G}\}$ is a base for a filter which we denote by $\mathcal{H}$.

We prove that $\mathcal{H}$ is a D-filter. Given any $H \in \mathcal{H}$, let $F \in \mathcal{F}$ and $G \in \mathcal{G}$ such that $F \oplus G \subset H$; take $F^{\prime}, F^{\prime \prime} \in \mathcal{F}$ satisfying (F1) and (F2) respectively, and choose $G^{\prime}, G^{\prime \prime} \in \mathcal{G}$ in a similar way. Clearly $H^{\prime}=F^{\prime} \oplus G^{\prime}$ and $H^{\prime \prime}=F^{\prime \prime} \oplus G^{\prime \prime}$ belong to $\mathcal{H}$; we show that $H^{\prime}$ satifies (F1) and $H^{\prime \prime}$ satisfies (F2) (with $H$ in place of $F$ ). If $a$ and $b$ are orthogonal elements of $H^{\prime}$, then $a=f_{1} \oplus g_{1}$ and $b=f_{2} \oplus g_{2}$, where $f_{1}, f_{2} \in F^{\prime}$ and $g_{1}, g_{2} \in G^{\prime}$; note that $f_{1} \perp f_{2}$ and $g_{1} \perp g_{2}$, hence $f=f_{1} \oplus f_{2} \in F$ and $g=g_{1} \oplus g_{2} \in G$ : therefore $a \oplus b=\left(f_{1} \oplus g_{1}\right) \oplus\left(f_{2} \oplus g_{2}\right)=\left(f_{1} \oplus f_{2}\right) \oplus\left(g_{1} \oplus g_{2}\right)=f \oplus g \in F \oplus G \subset H$. Now let $a \in H^{\prime \prime}$ and $c \in L$; let $f \in F^{\prime \prime}$ and $g \in G^{\prime \prime}$ such that $a=f \oplus g$, and put $d=(f \vee c) \ominus f$. We have $f^{\prime}=(f \vee c) \ominus c \in F$ and $g^{\prime}=(g \vee d) \ominus d \in G$. Since $g \vee d=g^{\prime} \oplus d$ and $f \vee c=f \oplus d$, applying 1.1(x) and 1.1(vii), we obtain $(a \vee c) \ominus c=(a \vee f \vee c) \ominus c=((f \oplus g) \vee(f \vee c)) \ominus c=$ $((f \oplus g) \vee(f \oplus d)) \ominus c=(f \oplus(g \vee d)) \ominus c=(f \oplus(g \vee d)) \ominus c=\left(f \oplus\left(g^{\prime} \oplus d\right)\right) \ominus c=\left((f \oplus d) \oplus g^{\prime}\right) \ominus c=$ $\left((f \vee c) \oplus g^{\prime}\right) \ominus c=((f \vee c) \ominus c) \oplus g^{\prime}=f^{\prime} \oplus g^{\prime} \in F \oplus G \subset H$.

It follows from $(2 ; 2)$ that both $\mathcal{F}$ and $\mathcal{G}$ are finer than $\mathcal{H}$. To complete the proof, consider any D-filter $\mathcal{H}^{\prime}$ such that both $\mathcal{F}$ and $\mathcal{G}$ are finer than $\mathcal{H}^{\prime}$ : we show that $\mathcal{H}^{\prime} \subseteq \mathcal{H}$. Let $H \in \mathcal{H}$ : by $(\mathrm{F} 1)$, there exists $H^{\prime} \in \mathcal{H}^{\prime}$ such that $H^{\prime} \oplus H^{\prime} \subseteq H$; since $H^{\prime} \in \mathcal{F} \cap \mathcal{G}$ we get $H^{\prime} \oplus H^{\prime} \in \mathcal{H}$ and hence $H \in \mathcal{H}$, too.

(b) Let $\mathcal{F}$ be the set of intersections of finite subsets of $\bigcup \Gamma$. We show that $\mathcal{F}$ is a filter.

Let $F_{1}, F_{2} \in \mathcal{F}$ : one has $F_{1}=\bigcap \mathcal{F}_{1}$ and $F_{1}=\bigcap \mathcal{F}_{1}$, where $\mathcal{F}_{1}$ and $\mathcal{F}_{2}$ are finite subsets of $\bigcup \Gamma$; if $G=F_{1} \cap F_{2}$, then $G \in \mathcal{F}$ because it is the intersection of $\mathcal{F}_{1} \cup \mathcal{F}_{2}$, which is again a finite subset of $\bigcup \Gamma$. Now let $F \in \mathcal{F}$ : then $F=\bigcap_{i=1}^{n} F_{i}$, where $F_{i} \in \mathcal{G}_{i}$ and $\mathcal{G}_{i} \in \Gamma$ for each $i \in\{1,2, \ldots, n\}$. If $G \supset F$, let $A=G \backslash F$; for each $i$, one has $G_{i}=A \cup F_{i} \in \mathcal{G}_{i}$, and $\bigcap_{i=1}^{n} G_{i}=\bigcap_{i=1}^{n}\left(A \cup F_{i}\right)=A \cup \bigcap_{i=1}^{n} F_{i}=A \cup F=G$ : hence $G \in \mathcal{F}$.

Now we check properties (F1) and (F2). Let $F \in \mathcal{F}$ : as above, $F=\bigcap_{i=1}^{n} F_{i}$, with $F_{i} \in \mathcal{G}_{i} \in \Gamma$. For each $i$, take $F_{i}^{\prime}$ and $G_{i}$ in $\mathcal{G}_{i}$ satisfying (F1) and (F2) respectively (with $F_{i}$ in place of $F$ ). Put $F^{\prime}=\bigcap_{i=1}^{n} F_{i}^{\prime}$ and $G=\bigcap_{i=1}^{n} G_{i}$ : clearly $F^{\prime}$ and $G$ belong to $\mathcal{F}$; we show that $F^{\prime}$ satifies (F1) and $G$ satisfies (F2). If $a$ and $b$ are orthogonal elements of $F^{\prime}$, then for each $i \in\{1,2, \ldots, n\}$ we have 
$a, b \in F_{i}^{\prime}$ and hence $a \oplus b \in F_{i}$ : therefore $a \oplus b \in F$. Similarly, if $a \in G$ and $c \in L$, then for each $i \in\{1,2, \ldots, n\}$ we have $a \in G_{i}$ and hence $(a \vee c) \ominus c \in F_{i}$ : therefore $(a \vee c) \ominus c \in F$.

Since it is clear that each $\mathcal{G} \in \Gamma$ is contained in $\mathcal{F}$ (indeed every $G$ in $\mathcal{G}$ is the intersection of $\{G\}$, which a finite subset of $\bigcup \Gamma$ ), it remains to prove that any D-filter which is finer than all filters in $\Gamma$ is finer than $\mathcal{F}$, too. So let $\mathcal{G}^{\prime} \in \mathcal{F} \mathcal{N} \mathcal{D}(L)$ such that $\mathcal{G} \subset \mathcal{G}^{\prime}$ for every $\mathcal{G} \in \Gamma$. Given $F \in \mathcal{F}$, one has $F=\bigcap_{i=1}^{n} F_{i}$ where $F_{i} \in \mathcal{G}_{i} \in \Gamma$, hence $F_{i} \in \mathcal{G}^{\prime}$, for each $i \in\{1,2, \ldots, n\}$; since $\mathcal{G}^{\prime}$ is a filter, we have $F \in \mathcal{G}^{\prime}$ : we conclude that $\mathcal{F} \subset \mathcal{G}^{\prime}$.

2.7. Corollary. $\mathcal{D U}(L)$ and $\mathcal{F} \mathcal{N D}(L)$ are distributive (complete) lattices.

Proof. $\quad$ By Theorem 2.4(c), it is enough to consider $\mathcal{F} \mathcal{N D}(L)$. Let $\mathcal{F}_{1}, \mathcal{F}_{2}$ and $\mathcal{G}$ be D-filters: we have to verify that $\left(\mathcal{F} \vee \mathcal{G}_{1}\right) \wedge\left(\mathcal{F} \vee \mathcal{G}_{2}\right) \subseteq \mathcal{F} \vee\left(\mathcal{G}_{1} \wedge \mathcal{G}_{2}\right)$

Given $H \in\left(\mathcal{F} \vee \mathcal{G}_{1}\right) \wedge\left(\mathcal{F} \vee \mathcal{G}_{2}\right)$, take $F_{1}, F_{2} \in \mathcal{F}$ and $G_{1}, G_{2} \in \mathcal{G}$ with $\left(F_{1} \cap G_{1}\right) \oplus\left(F_{2} \cap G_{2}\right) \subseteq H$; put $F=F_{1} \cap F_{2}$ and let $F^{\prime} \in \mathcal{F}$ satisfying 2.3(i). We complete the proof by showing that $F^{\prime} \cap\left(G_{1} \oplus G_{2}\right) \subseteq\left(F_{1} \cap G_{1}\right) \oplus\left(F_{2} \cap G_{2}\right)$.

Let $a \in F^{\prime} \cap\left(G_{1} \oplus G_{2}\right)$; choose $a_{1} \in G_{1}$ and $a_{2} \in G_{2}$ such that $a=a_{1} \oplus a_{2}$. Since $a_{1} \leq a$ and $a \in F^{\prime}$, one has $a_{1} \in F \subset F_{1}$ and hence $a_{1} \in F_{1} \cap G_{1}$; similarly one sees that $a_{2} \in F_{2} \cap G_{2}$. Therefore $a=a_{1} \oplus a_{2} \in\left(F_{1} \cap G_{1}\right) \oplus\left(F_{2} \cap G_{2}\right)$.

2.8. Proposition. If $\mathcal{F}, \mathcal{G} \in \mathcal{F} \mathcal{N D}(L)$, then $\{F \wedge G: F \in \mathcal{F}, G \in \mathcal{G}\}$ is a base for $\mathcal{F} \vee \mathcal{G}$, where $F \wedge G=\{f \wedge g: f \in F, g \in G\}$.

Proof. Given $F \in \mathcal{F}$ and $G \in \mathcal{G}$, since $F \cap G=\{a \wedge a: a \in F \cap G\} \subseteq\{f \wedge g: f \in F, g \in$ $G\}=F \wedge G$, it remains to prove that there exist $F^{\prime} \in \mathcal{F}$ and $G^{\prime} \in \mathcal{G}$ such that $F^{\prime} \wedge G^{\prime} \subseteq F \cap G$. Take $F^{\prime} \in \mathcal{F}$ satisfying 2.3(i), and let $G^{\prime}$ be a member of $\mathcal{G}$ satisfying 2.3(i) also, but with $G$ in place of $F$. If $f \in F^{\prime}$ and $g \in G^{\prime}$, then $f \wedge g \leq f$ hence $f \wedge g \in F$ and, similarly, $f \wedge g \leq g$ hence $f \wedge g \in G$ : therefore $f \wedge g \in F \cap G$.

\section{Generating D-uniformities by means of $k$-submeasures}

3.1. Definition. Let $k \geq 1$. We say that a function $\eta: L \rightarrow[0,+\infty]$ is a $k$-submeasure if the following conditions hold:

(S1) $\eta(0)=0$;

(S2) $\forall a, b \in L \quad[a \leq b \Longrightarrow \eta(a) \leq \eta(b)]$;

(S3) $\forall a, b \in L \quad[a \perp b \Longrightarrow \eta(a \oplus b) \leq k \eta(a)+\eta(b)]$;

(S4) $\forall a, b \in L \quad \eta((a \vee b) \ominus b) \leq k \eta(a)$.

A 1-submeasure is simply called a submeasure.

Observe that, if $L$ is an MV-algebra, then every function $\eta: L \rightarrow[0,+\infty]$ satisfying (S1), (S2) and (S3) with $k=1$ is a submeasure.

For every $\varepsilon>0$, put $S_{\varepsilon}=\{(x, y) \in[0,+\infty[\times[0,+\infty[:|x-y|<\varepsilon\} \cup\{(+\infty,+\infty)\}$. Then $\left\{S_{\varepsilon}: \varepsilon>0\right\}$ is base for a uniformity $\mathcal{S}$ on $[0,+\infty]$ whose relativization to $[0,+\infty$ [ is the usual uniformity, while $+\infty$ is a uniformly isolated point. In the sequel we will endow $[0,+\infty]$ with this uniformity.

3.2. Proposition. For every $k$-submeasure $\eta$ there exists a $D$-uniformity $\mathcal{U}(\eta)$ which is the weakest $D$-uniformity making $\eta$ uniformly continuous. 
Proof. For each $\varepsilon>0$, let $F_{\varepsilon}=\{a \in L: \eta(a)<\varepsilon\}$. Since $F_{\varepsilon_{1}} \cap F_{\varepsilon_{2}}=F_{\min \left\{\varepsilon_{1}, \varepsilon_{2}\right\}}$, the collection $\left\{F_{\varepsilon}: \varepsilon>0\right\}$ is a base for a filter $\mathcal{F}$. We show that $\mathcal{F}$ is a D-filter. Fix $F$ in $\mathcal{F}$, and take $\varepsilon>0$ with $F_{\varepsilon} \subset F$ : then $F^{\prime}=F_{\frac{\varepsilon}{k+1}}$ satisifies (F1) and $G=F_{\frac{\varepsilon}{k}}$ satisfies (F2).

From Theorem 2.4(b), the sets $F_{\varepsilon}^{\triangle}$ form a base for a D-uniformity $\mathcal{U}(\eta)$. Now we show that $\eta$ is $\mathcal{U}(\eta)$-uniformly continuous. Let $\varepsilon>0$ and choose $\delta=\frac{\varepsilon}{k}$; for every $(a, b) \in F_{\delta}^{\triangle}$, we have $\eta(a \vee b)=$ $\eta((a \triangle b) \oplus(a \wedge b)) \leq k \eta(a \triangle b)+\eta(a \wedge b)<\eta(a \wedge b)+k \delta=\eta(a \wedge b)+\varepsilon$. Thus, if $\eta(a \vee b)=+\infty$, then $\eta(a \wedge b)=+\infty$ whence, by monotonicity, $\eta(a)=\eta(b)=+\infty$; otherwise, again by monotonicity, $\eta(a)$ and $\eta(b)$ are both finite, and moreover $|\eta(a)-\eta(b)| \leq|\eta(a \vee b)-\eta(a \wedge b)|<\varepsilon$ : hence, in any case, $(\eta(a), \eta(b)) \in S_{\varepsilon}$.

Finally, let $\mathcal{V}$ be a D-uniformity on $L$ making $\eta$ uniformly continuous. We prove that $\mathcal{U}(\eta) \leq \mathcal{V}$, which, by Theorem 2.4(c), is equivalent to $\mathcal{F} \subset \mathcal{G}$, where $\mathcal{G}$ is the filter of neighbourhoods of 0 in $\mathcal{V}$. Take any $F \in \mathcal{F}$, and choose $\varepsilon>0$ with $F_{\varepsilon} \subset F$; since $\eta$ is continuous at 0 with respect to $\mathcal{V}$, and $\eta(0)=0$, there is some $G \in \mathcal{G}$ such that if $a \in G$ then $\eta(a)<\varepsilon$, i.e. $a \in F_{\varepsilon}$. It follows that $G \subset F_{\varepsilon} \subset F$, hence $F \in G$.

Our aim is to prove a sort of converse of the previous result, namely Theorem 3.4 below.

3.3. Proposition. Let $k, m \geq 1$, and $d$ be a pseudometric such that for all $a, b, c \in L$ :

(P1) $\quad d(a \wedge c, b \wedge c) \leq d(a, b)$;

(P2) $a \perp c, b \perp c \Longrightarrow d(a \oplus c, b \oplus c) \leq k d(a, b)$.

(P3) $\quad d((a \vee c) \ominus c,(b \vee c) \ominus c) \leq m d(a, b)$;

(P4) $\quad d((a \vee c) \ominus c, 0) \leq k d(a, 0)$;

For each $a \in L$, put $\tilde{\eta}(a)=d(a, 0)$. Then $\tilde{\eta}$ is $k$-submeasure and $U(\tilde{\eta})$ coincides with the uniformity induced by $d$.

Proof. It is clear that $\tilde{\eta}$ satisfies (S1). Moreover, if $a \leq b$, by (P1) we have $\tilde{\eta}(a)=d(a, 0)=$ $d(b \wedge a, 0 \wedge a) \leq d(b, 0)=\tilde{\eta}(b)$ and (S2) is proved. Now if $a, b \in L$ are orthogonal, then, applying the triangular inequality and (P2), we get $\tilde{\eta}(a \oplus b)=d(a \oplus b, 0) \leq d(a \oplus b, b)+d(b, 0) \leq k d(a, 0)+$ $d(b, 0)=k \tilde{\eta}(a)+\tilde{\eta}(b)$, that is (S3). Similarly, taking any $a, b \in L$, by (P4) we get $\tilde{\eta}((a \vee b) \ominus b)=$ $d((a \vee b) \ominus b, 0) \leq k d(a, 0)=k \tilde{\eta}(a)$, that is (S4).

Denote by $\mathcal{V}$ the uniformity induced by $d$ : the sets $V_{\varepsilon}=\{(a, b) \in L \times L: d(a, b)<\varepsilon\}$ form a base for $\mathcal{V}$, while the sets $F_{\varepsilon}^{\triangle}=\{(a, b) \in L \times L: \tilde{\eta}(a \triangle b)<\varepsilon\}$ form a base for $\mathcal{U}(\tilde{\eta})$, as we have seen in Proposition 3.2. We show that for every $\varepsilon>0$ there exists $\delta>0$ such that $F_{\delta}^{\triangle} \subseteq V_{\varepsilon}$ and $V_{\delta} \subset F_{\varepsilon}^{\triangle}$ : this will prove that $\mathcal{V}=\mathcal{U}(\tilde{\eta})$.

Take $\delta=\frac{\varepsilon}{2 k m}$. Given $(a, b) \in F_{\delta}^{\triangle}$, applying (P1) and (P2), we have $d(a, b) \leq d(a, a \wedge$ $b)+d(a \wedge b, b)=d((a \vee b) \wedge a,(a \wedge b) \wedge a)+d((a \wedge b) \wedge b,(a \vee b) \wedge b) \leq 2 d(a \vee b, a \wedge b)=$ $2 d((a \triangle b) \oplus(a \wedge b), 0 \oplus(a \wedge b)) \leq 2 k d(a \triangle b, 0)=2 k \tilde{\eta}(a \triangle b)<2 k \delta \leq \varepsilon$, so that $(a, b) \in V_{\varepsilon}$. Therefore $F_{\delta}^{\triangle} \subseteq V_{\varepsilon}$.

Now let $(a, \bar{b}) \in V_{\delta}$. Recall that, by 1.1(ii), $(a \triangle b) \ominus((a \vee b) \ominus a)=a \ominus(a \wedge b)$ and, by 1.1(viii), $(a \ominus(a \wedge b)) \wedge(b \ominus(a \wedge b))=0$. Hence, applying first the triangle inequality and then (P2), (P3), (P1) and again (P3), we obtain $\tilde{\eta}(a \triangle b)=d(a \triangle b, 0) \leq d(a \triangle b,(a \vee b) \ominus a)+d((a \vee b) \ominus a, 0)=$ $d(((a \vee b) \ominus a) \oplus(a \ominus(a \wedge b)),(a \vee b) \ominus a)+d((a \vee b) \ominus a,(a \vee a) \ominus a) \leq k d(a \ominus(a \wedge b), 0)+m d(a, b)=$ $k d((a \ominus(a \wedge b)) \wedge(a \ominus(a \wedge b)),(b \ominus(a \wedge b)) \wedge(a \ominus(a \wedge b)))+m d(a, b) \leq k d(a \ominus(a \wedge b), b \ominus(a \wedge$ $b))+m d(a, b) \leq k m d(a, b)+m d(a, b)<(k+1) m \delta \leq \varepsilon$ so that $(a, b) \in F_{\varepsilon}^{\triangle}$. We conclude that $V_{\delta} \subset F_{\varepsilon}^{\triangle}$.

Recall that if $\mathbb{G}$ is a topological Abelian group, then a mapping $\mu: L \rightarrow \mathbb{G}$ is called a modular measure if the following hold, for all $a, b \in L$ :

(M1) $\mu(a)+\mu(b)=\mu(a \vee b)+\mu(a \wedge b)$.

(M2) If $a \perp b$, then $\mu(a \oplus b)=\mu(a)+\mu(b)$. 
Moreover, (see [1, Theor. 3.2]) the sets $\{(a, b) \in L \times L: \forall r \leq a \triangle b \quad \mu(r) \in W\}$, where $W$ is a neighbourhood of 0 in $\mathbb{G}$, form a base for a D-uniformity $\mathcal{U}$; this $\mathcal{U}$ is called the D-uniformity generated by $\mu$. Note that, in case $\mu$ is positive real-valued (hence in particular a submeasure), $\mathcal{U}$ agrees with the $\mathcal{U}(\mu)$ constructed in Proposition 3.2.

3.4. Theorem. Let $U$ be a D-uniformity on $L$. Then:

(a) For every $k>1$ there is a family $\left\{\tilde{\eta}_{\lambda}\right\}_{\lambda \in \Lambda}$ of $k$-submeasures with $\mathcal{U}=\sup _{\lambda \in \Lambda} \mathcal{U}\left(\tilde{\eta}_{\lambda}\right)$. Moreover, if $\mathcal{U}$ has a countable base, we can choose $|\Lambda|=1$.

(b) If $\mathcal{U}$ is generated by a modular measure $\mu: L \rightarrow \mathbb{G}$, where $\mathbb{G}$ is a topological Abelian group, then there is a family $\left\{\tilde{\eta}_{\lambda}\right\}_{\lambda \in \Lambda}$ of submeasures with $\mathcal{U}=\sup _{\lambda \in \Lambda} \mathcal{U}\left(\tilde{\eta}_{\lambda}\right)$.

(c) If $L$ is an $M V$-algebra, there is a family $\left\{\tilde{\eta}_{\lambda}\right\}_{\lambda \in \Lambda}$ of submeasures with $\mathcal{U}=\sup _{\lambda \in \Lambda} \mathcal{U}\left(\tilde{\eta}_{\lambda}\right)$. Proof.

(a) For every $a, b \in L$, put $f(a, b)=a \wedge b, g(a, b)=\left(a \wedge b^{\perp}\right) \oplus b$ and $h(a, b)=(a \vee b) \ominus b$. By [15, Prop. 1.1(b)], $\mathcal{U}$ has base consisting of sets $U$ such that, for every $\left(a, a^{\prime}\right) \in U$ and every $b \in L,\left(f(a, b), f\left(a^{\prime}, b\right)\right)=\left(f(b, a), f\left(b, a^{\prime}\right)\right) \in U$; since $g$ and $h$ are $\mathcal{U}$-uniformly continuous, from [15, Prop. 1.2] it follows that $\mathcal{U}$ is generated by a family $\left\{d_{\lambda}\right\}_{\lambda \in \Lambda}$ of pseudometrics (a single pseudometric if $\Lambda$ is countable) such that, for every $\lambda \in \Lambda$ and all $a, a^{\prime}, b, b^{\prime} \in L$ :

$$
\begin{aligned}
& d_{\lambda}\left(f(a, b), f\left(a^{\prime}, b^{\prime}\right)\right) \leq d_{\lambda}\left(a, a^{\prime}\right)+d_{\lambda}\left(b, b^{\prime}\right), \\
& d_{\lambda}\left(g(a, b), g\left(a^{\prime}, b^{\prime}\right)\right) \leq k\left(d_{\lambda}\left(a, a^{\prime}\right)+d_{\lambda}\left(b, b^{\prime}\right)\right), \\
& d_{\lambda}\left(h(a, b), h\left(a^{\prime}, b^{\prime}\right)\right) \leq k\left(d_{\lambda}\left(a, a^{\prime}\right)+d_{\lambda}\left(b, b^{\prime}\right)\right) .
\end{aligned}
$$

Clearly each $d_{\lambda}$ satisfies (P1) and (P2), as well as (P3) with $m=k$, hence also (P4). Therefore, applying Proposition 3.3, the conclusion follows.

(b) Let $\left\{p_{\lambda}\right\}_{\lambda \in \Lambda}$ be a family of group seminorms generating the topology of $\mathbb{G}$. By [12, Theor. 3], $\mathcal{U}$ is generated by the family of pseudometrics $\left\{d_{\lambda}\right\}_{\lambda \in \Lambda}$ where, for every $\lambda \in \Lambda$,

$$
d_{\lambda}(a, b)=\sup \left\{p_{\lambda}(\mu(r)-\mu(s)): r, s \in[a \wedge b, a \vee b]\right\} ;
$$

moreover $d_{\lambda}$ satisifies (P1) and the following:

$$
\forall a, b, c \in L \quad d_{\lambda}(a \vee c, b \vee c) \leq d_{\lambda}(a, b) .
$$

We can complete the proof, applying Proposition 3.3, once we have shown that each $d_{\lambda}$ satisfies both (P2) and (P3) with $m=k=1$, hence also (P4).

Fix $\lambda \in \Lambda$. Given $a, b \in L$, observe first that $d_{\lambda}(a, b)=\sup \left\{p_{\lambda}(\mu(r)): r \leq a \triangle b\right\}$. Now let $c \in L$; by Lemma 2.2 we have $((a \vee c) \ominus c) \triangle((b \vee c) \ominus c)=(a \vee c) \triangle(b \vee c)$; therefore, by $(3 ; 1)$, $d_{\lambda}((a \vee c) \ominus c),((b \vee c) \ominus c)=d_{\lambda}(a \vee c, b \vee c) \leq d_{\lambda}(a, b)$. Finally, if $c \perp a$ and $c \perp b$, then, again by 2.2 , we have $(a \oplus c) \triangle(b \oplus c)=a \triangle b$; hence $d_{\lambda}(a \oplus c, b \oplus c)=d_{\lambda}(a, b)$.

(c) Define $f, g$ and $h$ as in the proof of (a). By [15, Prop. 1.5], since $g$ is associative and distributive with respect to $f$, the uniformity $\mathcal{U}$ has base consisting of sets $U$ such that, for every $\left(a, a^{\prime}\right) \in U$ and every $b \in L,\left(f(a, b), f\left(a^{\prime}, b\right)\right)=\left(f(b, a), f\left(b, a^{\prime}\right)\right) \in U$ and $\left(g(a, b), g\left(a^{\prime}, b\right)\right)=$ $\left(g(b, a), g\left(b, a^{\prime}\right)\right) \in U$; moreover $h$ is $\mathcal{U}$-uniformly continuous, and therefore from [15, Prop. 1.2] it follows that, for any $m>1, \mathcal{U}$ is generated by a family $\left\{d_{\lambda}\right\}_{\lambda \in \Lambda}$ of pseudometrics (a single pseudometric if $\Lambda$ is countable) such that, for every $\lambda \in \Lambda$ and all $a, a^{\prime}, b, b^{\prime} \in L$ :

$$
\begin{aligned}
& d_{\lambda}\left(f(a, b), f\left(a^{\prime}, b^{\prime}\right)\right) \leq d_{\lambda}\left(a, a^{\prime}\right)+d_{\lambda}\left(b, b^{\prime}\right), \\
& d_{\lambda}\left(g(a, b), g\left(a^{\prime}, b^{\prime}\right)\right) \leq\left(d_{\lambda}\left(a, a^{\prime}\right)+d_{\lambda}\left(b, b^{\prime}\right)\right), \\
& d_{\lambda}\left(h(a, b), h\left(a^{\prime}, b^{\prime}\right)\right) \leq m\left(d_{\lambda}\left(a, a^{\prime}\right)+d_{\lambda}\left(b, b^{\prime}\right)\right) .
\end{aligned}
$$

Clearly each $d_{\lambda}$ satisfies $(\mathrm{P} 1),(\mathrm{P} 2)$ with $k=1$ and $(\mathrm{P} 3)$; it remains to show that $(\mathrm{P} 4)$ with $k=1$ is satisified, too. Let $a, c \in L$ : by $(\mathrm{P} 1)$, we have $d_{\lambda}((a \vee c) \ominus c, 0)=d_{\lambda}(a \ominus(a \wedge c), 0)=$ $d_{\lambda}\left((a \wedge(a \ominus(a \wedge c)), 0 \wedge(a \ominus(a \wedge c))) \leq d_{\lambda}(a, 0)\right.$.

The reader should note that 3.4(c) was already proved in [6, Theor. 2.5]. 


\section{References}

[1] A. Avallone. Lattice uniformities on orthomodular structures. Mathematica Slovaca, 51:403-419, 2001.

[2] A. Avallone, G. Barbieri, and P. Vitolo. Hahn decomposition of modular measures and applications. Preprint.

[3] A. Avallone and A. Basile. On a Marinacci uniqueness theorem for measures. Preprint.

[4] A. Avallone and P. Vitolo. Congruences and ideals of effect algebras. Preprint.

[5] A. Avallone and H. Weber. Lattice uniformities generated by filters. Journal of Mathematical Analysis and Applications, 209(2):507-528, 1997.

[6] G. Barbieri and H. Weber. A topological approach to the study of fuzzy measures. In Functional Analysis and Economic Theory, Samos, 1996, pages 17-46. Springer Verlag, Berlin, 1998.

[7] E. G. Beltrametti and G. Cassinelli. The Logic of Quantum Mechanics. Addison-Wesley Publ. Co., Reading, Mass., 1981.

[8] M. K. Bennet and D. J. Foulis. Effect algebras and unsharp quantum logics. Found. Phys., 24(10):1331-1352, 1994.

[9] D. Butnariu and P. Klement. Triangular Norm-based Measures and Games with Fuzzy Coalitions. Kluwer Academic Publishers, Dordrecht, 1993.

[10] A. Dvurečenskij and S. Pulmannová. New Trends in Quantum Structures. Kluwer Academic Publishers, Dordrecht, 2000.

[11] L. G. Epstein and J. Zhang. Subjective probabilities on subjectively unamibguous events. Econometrica, 69(2):265-306, 2001.

[12] I. Fleischer and T. Traynor. Group-valued modular functions. Algebra Universalis, 14:287-291, 1982.

[13] M. G. Graziano. Uniformities of Fréchet-Nikodym type on Vitali spaces. Semigroup Forum, 61(1):91$115,2000$.

[14] H. Weber. Uniform lattices. I: A generalization of topological Riesz spaces and topological Boolean rings. Annali di Matematica Pura e Applicata (4), 160:347-370, 1991.

[15] H. Weber. Metrization of uniform lattices. Czechoslovak Mathematical Journal, 118:271-280, 1993.

[16] H. Weber. Lattice uniformities and modular functions on orthomodular lattices. Order, 12:295-305, 1995. 